\title{
Serial plasma adrenaline and noradrenaline levels in myocardial infarction using a new double isotope technique
}

\author{
David C. Siggers, Cheryl Salter, and David C. Fluck \\ From the Department of Cardiology, Guy's Hospital, London S.E.I
}

\begin{abstract}
The plasma levels of adrenaline and noradrenaline have been measured in 10 patients with myocardial infarction using a new double isotope dilution technique. Adrenaline and noradrenaline levels were measured on admission and at 12-hourly intervals for the next 5 days. There were significant correlations between raised noradrenaline and (I) raised systolic blood pressure, (2) the presence of pulmonary oedema, and (3) transfer of patients back to the general wards; and between raised adrenaline levels and (I) the presence of pulmonary oedema, and (2) the presence of ventricular ectopics. The results suggest that emotional factors play a part in the increase in catecholamine levels in these patients and that adequate sedation may result in lower levels. One patient with cardiac arrest followed by complete heart block and cardiogenic shock had particularly high catecholamine levels, exceeding those observed in a case of phaeochromocytoma.
\end{abstract}

The study of the activity of the sympathetic nervous system and adrenal medulla in patients with myocardial infarction has been hampered by the difficulty of measuring, accurately, free adrenaline and noradrenaline levels in plasma. Until recently the methods for measuring these amines have relied on their properties of fluorescence under ultraviolet light. Workers who have used these techniques have often found accurate results hard to obtain at the low levels of catecholamines present in normal plasma (Crout, 1959; Udenfriend, 1959). In addition, these methods do not employ internal standardization for each individual sample and values for adrenaline must be derived from total catecholamine and noradrenaline values rather than being measured directly. There are, as has been shown recently by Carruthers $e t$ al. (1970), several common dietary and therapeutic agents (including tea, coffee, ampicillin, vitamins, aminophylline, and lasix) which cause moderate or striking fluorescence in vitro when put through the same extraction and analysis stages as the plasma samples.

A new approach to this problem which has overcome these difficulties has been the application of the double isotope dilution technique by Engelman, Portnoy, and Lovenberg (I968). Recently this method has been

Received 25 February 1971. adapted (Siggers, Salter, and Toseland, 1970) to allow differential measurement of adrenaline and noradrenaline in plasma as well as total catecholamine levels.

Increased levels of catecholamines in the urine after myocardial infarction have been reported by many authors (Forssman, Hansson, and Jensen, 1952; Nuzum and Bischoff, 1953; Valori, Thomas, and Shillingford, 1967; Januszewicz et al., 1968; Wallace, I968; Jewitt et al., 1969). In particular, high levels appeared to be associated with heart failure, cardiogenic shock, and arrhythmias. There have been previous reports of plasma catecholamine levels following myocardial infarction, using fluorimetric techniques (Gazes, Richardson, and Woods, 1959; McDonald et al., 1969). Gazes et al. (1959) reported a single measurement within the first 36 hours and a second measurement at 72 hours after admission. The noradrenaline levels were raised in all cases and the adrenaline in about half the cases. McDonald et al. (1969) measured the plasma levels on one occasion, usually within 24 hours of admission, and found raised noradrenaline levels. There was an association between high noradrenaline levels and atrial and early ventricular arrhythmias.

In view of the problems inherent in fluorescent methods and the points raised recently by Carruthers et al. (1970), it is difficult to 
interpret previous measurements of plasma catecholamines after myocardial infarction. In this study we have made serial measurements of plasma adrenaline and noradrenaline levels, using the double isotope dilution method, in patients who have been admitted with myocardial infarction, and we have correlated these findings with the clinical information obtained in each case.

\section{Method}

Blood samples, for free adrenaline and noradrenaline estimation, were taken on admission to the intensive care unit and at 12-hourly intervals ( 9 am and $9 \mathrm{pm}$ ) for 5 days. All patients were admitted to the intensive care unit where electrocardiogram, blood pressure, pulse rate, and central venous pressure were monitored. A record was made each day of the presence or absence of heart failure and of radiological pulmonary oedema, the plasma levels of alphahydroxybutyric dehydrogenase and aspartate aminotransferase; blood gases, electrolytes, and urea; the urine output; the sodium, potassium, and urea content of the urine and its osmolality. A record was made of the details of any arrhythmia and any antiarrhythmic drug given. All other drugs were also recorded and a record was made of the patient's psychological state during the first 5 days. In addition to the patients with acute myocardial infarction, blood levels of adrenaline and noradrenaline were measured in ro normal volunteers and in one patient with a phaeochromocytoma.

Blood samples $(20 \mathrm{ml})$ were taken by venepuncture into a siliconized syringe and transferred to a tube containing sodium citrate and ascorbic acid. The tube was kept in ice and spun down within ro minutes in a refrigerated centrifuge to separate the plasma. Small quantities of tritiumlabelled adrenaline and tritium-labelled noradrenaline were added to each sample as an internal standard. Plasma samples ( $10 \mathrm{ml}$ ) were passed through a cation exchange column and then incubated with the enzyme catechol-ortho-methyl transferase together with a ${ }^{14} \mathrm{C}$-labelled methyl donor (adenosyl methionine) to convert them to the ${ }^{14} \mathrm{C}$-methyl derivatives: ${ }^{14} \mathrm{C}$ metadrenaline and ${ }^{14} \mathrm{C}$ normetadrenaline. The enzyme reaction was stopped and $20 \mu \mathrm{g}$ each of unlabelled metadrenaline and normetadrenaline were added as carriers and as markers for the subsequent electrophoresis. The methylcatecholamines were extracted in ether (Anton and Sayre, 1966) and subjected to electrophoresis at 500 volts on a thin layer of cellulose to separate the metadrenaline from the normetadrenaline. These substances were dyed with a p-nitraniline spray. Each spot was transferred into a vial together with toluene scintillant and thixotrophic gel. The vials were placed in a scintillation counter and counted for tritium and ${ }^{14} \mathrm{C}$. The percentage recovery of each individual sample was known from the tritium count, and, by the ratio of ${ }^{14} \mathrm{C}$ to tritium, the levels of noradrenaline and adrenaline in the original sample were calculated.
Recoveries usually ranged from to to 90 per cent but occasionally samples gave a very poor recovery and could not be used for calculations. The plasma catecholamine levels in the first patient measured were not differentiated into adrenaline and noradrenaline.

\section{Patients}

Ten patients, admitted with acute myocardial infarction, were selected randomly for this study. Nine patients were male and one was female. The ages ranged from 47 to 68 years, with a mean age of 59 years. The diagnosis of acute myocardial infarction was made on the usual electrocardiographic criteria together with an increase in the serum levels of myocardial enzymes in 9 patients. In one patient, however, the electrocardiogram showed changes of an old posterior infarct, and the enzyme levels did not rise. However, the clinical history and course were highly suggestive of a recent myocardial infarction.

\section{Results}

The mean noradrenaline and adrenaline levels for the nine patients are shown in Fig. I and 2. The upper limit of the normal levels based on a series of blood samples taken from ro normal volunteers was found to be $0.60 \mu \mathrm{g} / \mathrm{l}$. for noradrenaline and $0.25 \mu \mathrm{g} / \mathrm{l}$. for adrenaline. On admission the levels were above normal but by the final sample on the fifth day they had returned to normal. There was a rise in both adrenaline and noradrenaline on the fourth day.

There was a significant linear correlation between raised noradrenaline levels and systolic blood pressure (correlation coefficient $0.323 \mathrm{P}<0.02$ ) but not between adrenaline and systolic blood pressure. No significant linear correlation was found between either adrenaline or noradrenaline and pulse rate. One patient who developed complete heart block had very high levels of catecholamines, particularly when the ventricular rate was not being maintained with a pacemaker (Fig. 3). On the other hand, a patient who developed sinus bradycardia was found to have normal levels initially, though one other patient with normal levels on admission did not have a slow heart rate. Patients who developed ventricular ectopics had significantly higher peak levels of adrenaline $(P<0.02)$, but not noradrenaline, than those with no observed ventricular ectopics. However, when all levels of both noradrenaline and adrenaline were analysed they were not significantly higher in the presence of ventricular ectopics (Table I). The presence of pulmonary oedema was associated with significantly higher levels of both adrenaline $(P<0.005)$ and noradrenaline $(\mathbf{P}<0.05)$. However, peak values of adrenal- 


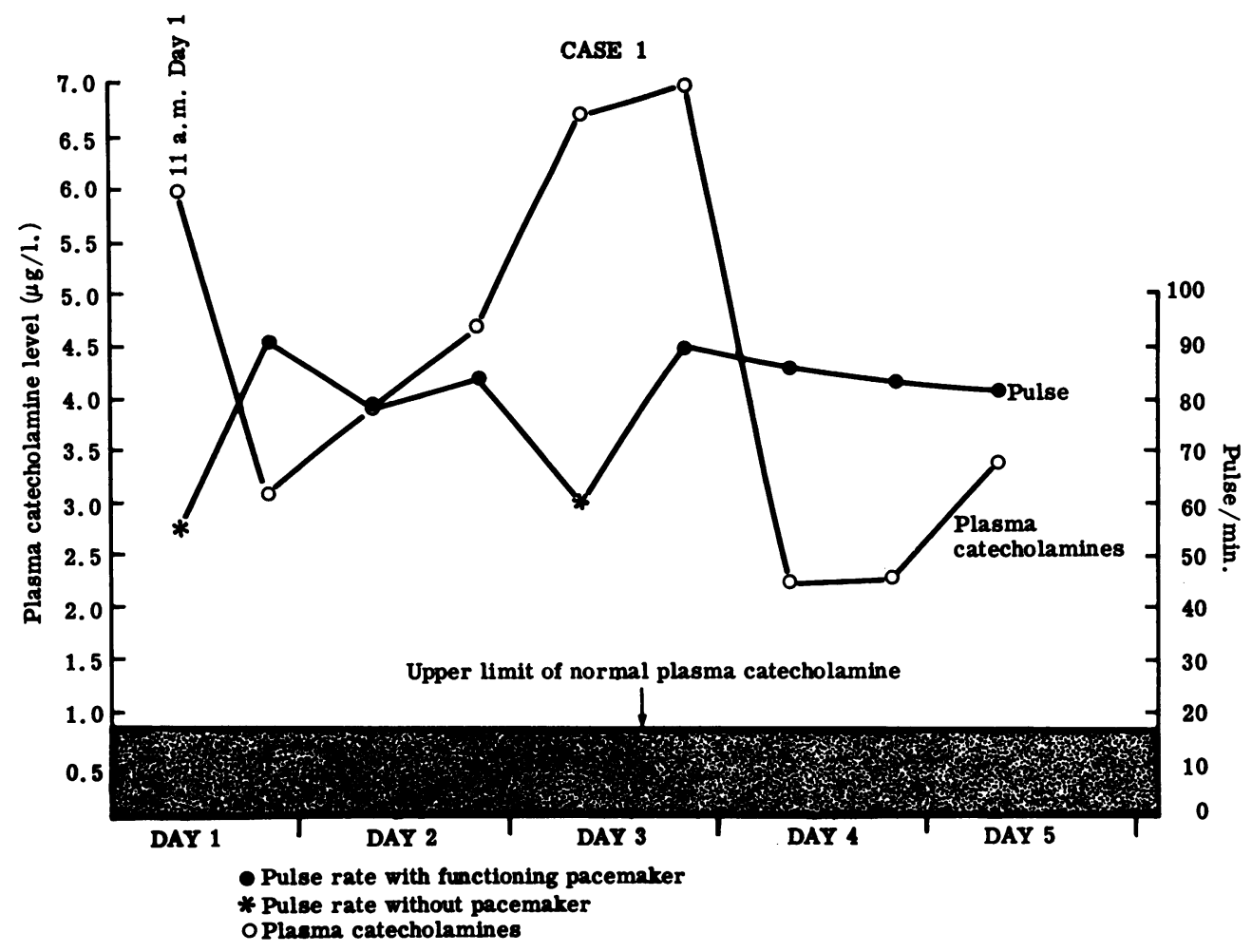

FIG. I The mean levels ( \pm standard error) of plasma adrenaline for 9 patients during the first 5 days of admission.

FIG. 2 The mean levels ( \pm standard error) of plasma noradrenaline for 9 patients during the first 5 days of admission.

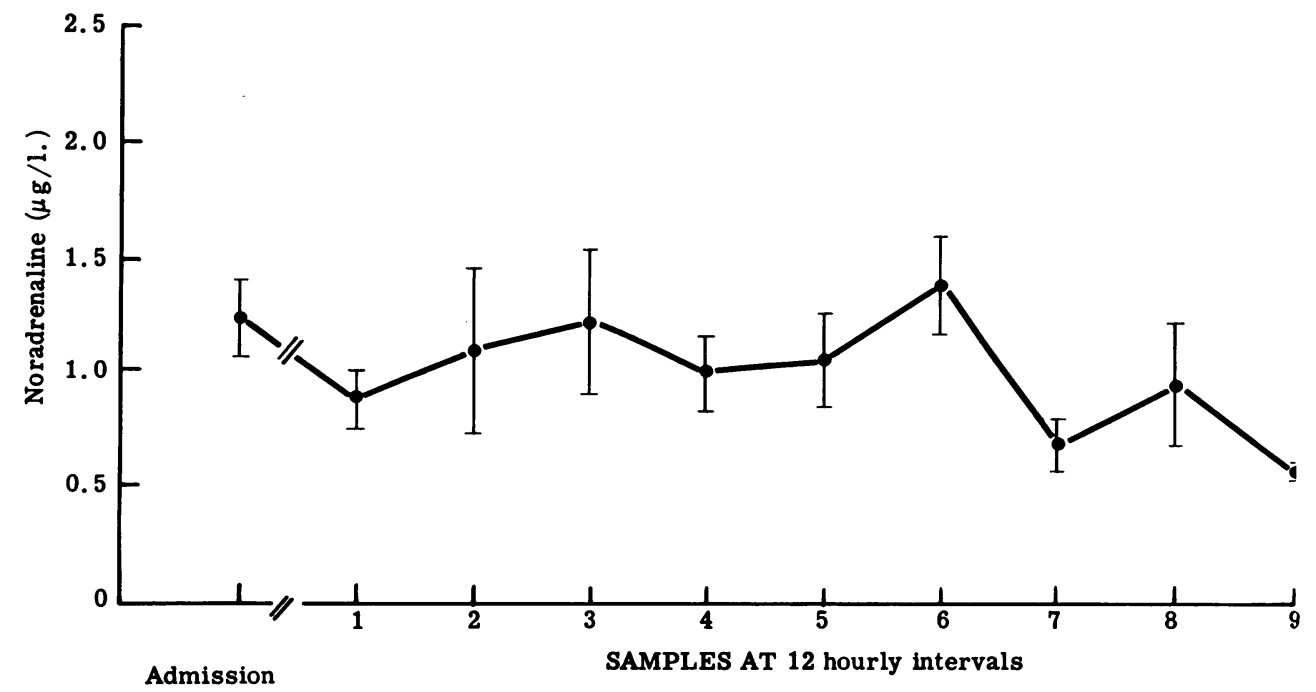


TABLE I

\begin{tabular}{lcc}
\hline Clinical situation & Noradrenaline & Adrenaline \\
\hline a) Mean values ( \pm standard error) of all plasma nor- \\
\multicolumn{2}{c}{ adrenaline and adrenaline levels } \\
\hline Pulmonary oedema & $\mathrm{I} \cdot 296 \pm 0.164$ & $0.878 \pm 0.139$ \\
No pulmonary oedema & $0.928 \pm 0.086$ & $0.483 \pm 0.052$ \\
Ventricular ectopics & $\mathrm{I} \cdot 100 \pm 0.166$ & $0.651 \pm 0.149$ \\
No arrhythmia & $0.999 \pm 0.087$ & $0.554 \pm 0.060$
\end{tabular}

b) Mean peak values ( \pm standard error) of plasma noradrenaline and adrenaline

\begin{tabular}{lll}
\hline Pulmonary oedema & $\mathbf{1} \cdot 632 \pm 0.293$ & $0.990 \pm 0.075$ \\
No pulmonary oedema & $\mathbf{I} \cdot 587 \pm 0.245$ & $1 \cdot 112 \pm 0.178$ \\
Ventricular ectopics & $\mathbf{I} \cdot 880 \pm 0.229$ & $\mathrm{I} \cdot 390 \pm 0.123$ \\
No arrhythmia & $\mathbf{I} 478 \pm 0.214$ & $0.966 \pm 0.067$ \\
\hline
\end{tabular}

c) Mean values ( \pm standard error) of plasma noradrenaline levels before and after transfer to general wards

\begin{tabular}{lll}
\hline Before & $0.681 \pm 0.103$ & $0.391 \pm 0.118$ \\
After & $1 \cdot 106 \pm 0.224$ & $0.514 \pm 0.147$ \\
\hline
\end{tabular}

ine and noradrenaline in patients with pulmonary oedema were not significantly different from those without, though it must be appreciated the numbers were small for statistical analysis (Table I).

The first blood sample taken after return to the general ward from the intensive care unit showed a significantly higher mean level of noradrenaline $(P<0.05)$ than the sample taken immediately before transfer. Mean adrenaline levels were also higher but not
TABLE 2 Plasma noradrenaline and adrenaline levels in case of phaeochromocytoma

\begin{tabular}{lll}
\hline & Sample I & Sample 2 \\
\hline Noradrenaline $\mu \mathrm{g} /$ litre & 2.1 & 4.3 \\
Adrenaline $\mu \mathrm{g} /$ litre & 0.5 & 0.8 \\
\hline
\end{tabular}

significantly so (Fig. 4 and Table I). Plasma noradrenaline and adrenaline levels in a patient with a phaeochromocytoma are shown (Table 2) for comparison with the levels of plasma catecholamines after myocardial infarction, and it can be seen that in one patient with acute myocardial infarction (Fig. 3) the levels of catecholamines even exceeded these values.

\section{Discussion}

The new isotope dilution method for differential estimation of plasma adrenaline and noradrenaline gives a specific separation of the two amines and an accurate recovery value for each sample. There is no interference from fluorescent substances in the plasma.

A recent study (Carruthers et al., 1970) reported that venepuncture did not cause a significant increase in noradrenaline levels, but suggested there was a significant increase in adrenaline levels. The method used for that study, however, relies on derived values for adrenaline and not on direct measurements making the values 'much less dependable' (Carruthers et al., 1970). On admission the mean noradrenaline level for the 9 patients

FIG. 3 Levels of plasma catecholamines (adrenaline and noradrenaline combined) in a patient who developed complete heart block.

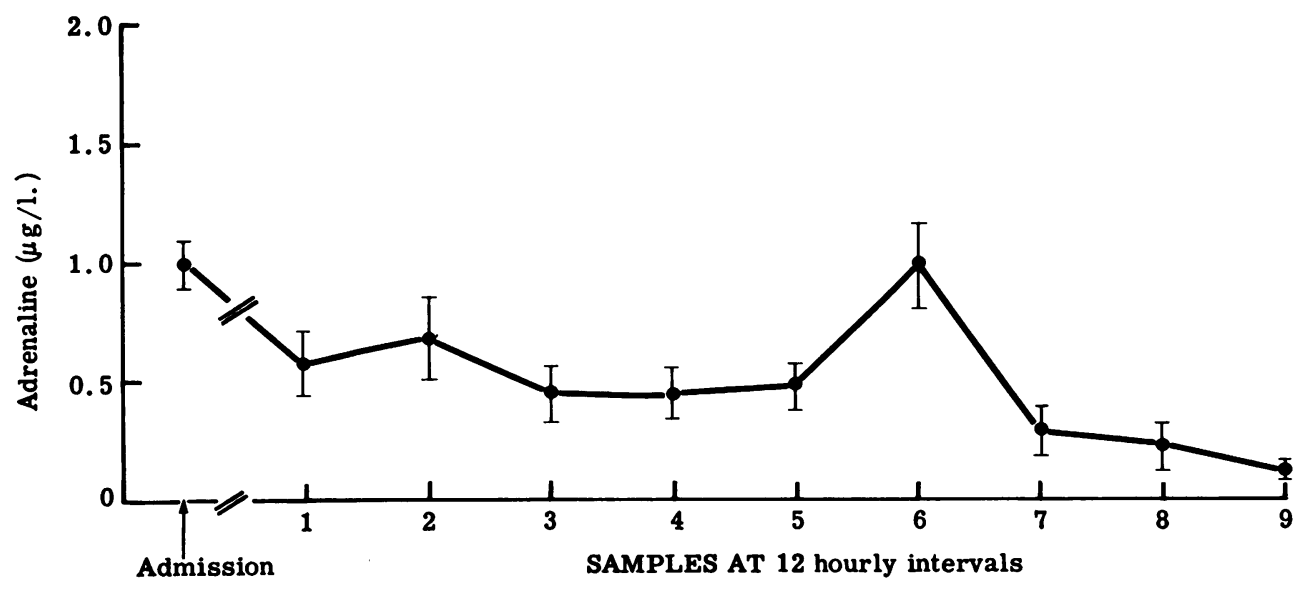


in this study was raised to over twice the upper limit of normal, while the mean adrenaline level was raised to about four times the upper limit of normal. This in general confirms the findings of earlier workers (Gazes et al., I959; Valori et al., 1967; Jewitt et al., 1969) though McDonald et al. (1969) found no increase in adrenaline levels.

It is of interest that the catecholamine levels tended to rise after transfer to the general ward. This may have been related to the fact that sedation of these patients was greater while in the intensive care unit. It suggests that emotional factors may play a part in the raised catecholamines in these patients. McDonald et al. (1969) concluded that stress did not play an important part in the increase of plasma catecholamines because patients undergoing cardiac catheterization showed no increase; perhaps the cardiac catheter patients were sedated or expertly reassured. Vendsalu (1960) showed that catecholamine levels increased when his subjects changed to the head-up tilt position from the recumbent position, and it is possible that withdrawal of sedation from our patients tended to encourage them to adopt a sitting position in bed instead of a recumbent one. This work (Vendsalu, I960), however, was carried out Io years ago by an earlier fluorescent method and needs re-examination.

The relation between blood pressure and noradrenaline level is complex. Valori et al. (1967) found no clear correlation, since patients with normal arterial pressures had either normal or raised urinary catecholamines; patients with low arterial pressures also had variable catecholamine levels but those with more severe degrees of heart failure had the higher catecholamine levels. The patient with episodes of hypotension and bradycardia related to complete heart block had very high plasma catecholamine levels related to these episodes, while at other times, when blood pressure and pulse rate were supported at normal levels by an endocardial pacemaker, the catecholamine levels were not as high though they were still raised above normal (Fig. 3). In the other 9 patients, none of whom was hypotensive, there was a correlation between raised systolic blood pressure and increased noradrenaline levels. In another patient the level of plasma noradrenaline taken half an hour before an episode of sinus bradycardia was $0.6 \mu \mathrm{g} / \mathrm{l}$. and of adrenaline was $0.2 \mathrm{ug} / 1$. Jewitt et al. (1969) also found normal catecholamine levels in 24-hour urine collections in relation to sinus bradycardia treated with atropine, and Valori et al. (1967) found normal catecholamine levels in 24 -hour
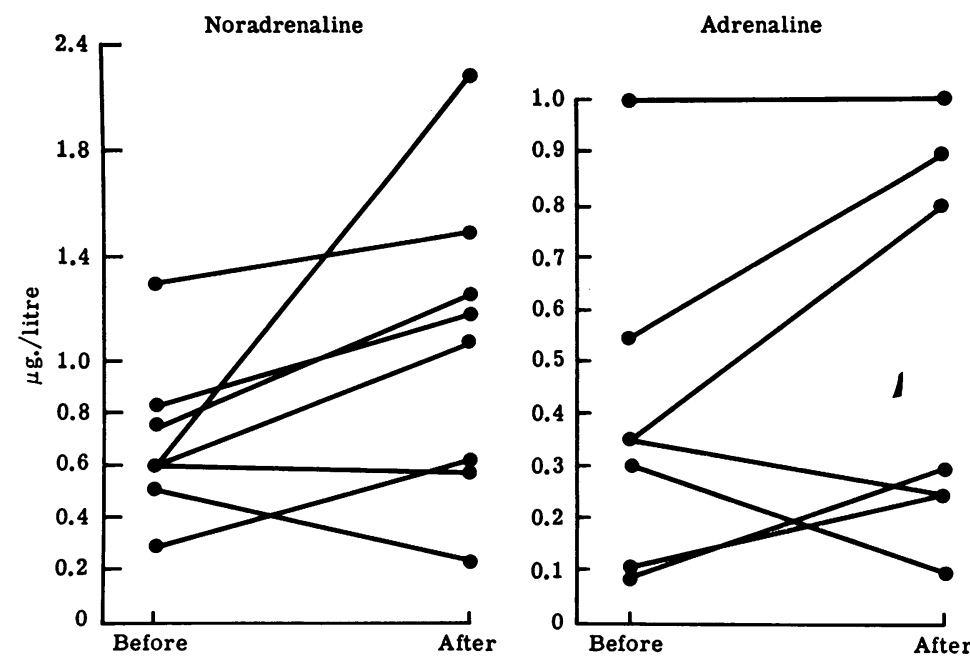

FIG. 4 Left: Plasma noradrenaline levels taken before and after transfer from the intensive care unit to the general ward. There is a significant increase $(P<0.05)$ in these levels after transfer. Right: plasma adrenaline levels taken before and after transfer from the intensive care unit to the general ward. There was no significant difference in the levels.

urine samples in relation to continued bradycardia and hypotension. Possibly the absence of increased catecholamine levels may be related to the absence of left ventricular failure in patients who develop sinus bradycardia and may even be a predisposing factor to the development of vasovagal episodes which are likely to occur in these patients (Fluck et al., 1967). An association between congestive cardiac failure, in the absence of myocardial infarction and raised urinary excretion of catecholamines, was shown by Chidsey, Braunwald, and Morrow (1965). Valori et al. (1967) and Jewitt et al. (1969) found high levels of urinary catecholamines in pulmonary oedema and increasing levels with increasing severity of heart failure. We found significantly higher noradrenaline $(P<0.05)$ and adrenaline $(\mathbf{P}<0.005)$ levels with pulmonary oedema. However, mean peak levels of both adrenaline and noradrenaline in patients with pulmonary oedema were little different from those in patients without pulmonary oedema, but it must be remembered that the number of patients studied was not large. The patient illustrated in Fig. 3 had the features of severe congestive heart failure on two occasions, when in complete heart block unsupported by a pacemaker, and the plasma catecholamine 
levels on these occasions were the highest recorded in the series. The first of these two episodes was within two hours after a cardiac arrest in ventricular fibrillation when the plasma catecholamine level was $6 \cdot 0 \mu \mathrm{g} / \mathrm{l}$. Very high catecholamine production occurs in the most severe forms of heart failure, and it may be that the heart failure itself is a stimulus to catecholamine production, perhaps by provoking peripheral rather than adrenal release. There was some relation between catecholamines and arrhythmias. As previously mentioned, the one patient who was seriously ill with complete heart block and episodes of ventricular fibrillation had very high levels. Patients who developed ventricular ectopics were found to have significantly higher peak levels of adrenaline, but not noradrenaline, than those who did not. The question whether high levels of catecholamines are desirable or undesirable in myocardial infarction cannot be answered by this study; the clinical events associated with high levels may not be the cause of them but may result from them.

The results of the present study may be useful in suggesting that adequate sedation may be of value in lowering catecholamine levels. It is possible that patients with high catecholamine levels without severe heart failure or hypotension may benefit from sympathetic blockage. Conversely, it may be inferred that patients without raised levels are those likely to have sinus bradycardia.

\section{References}

Anton, A. H., and Sayre, D. F. (1966). Distribution of metanephrine and normetanephrine in various animals and their analysis in diverse biologic material. Fournal of Pharmacology and Experimental Therapeutics, 153, 15 .

Carruthers, M., Taggart, P., Conway, N., Bates, D., and Somerville, W. (1970). Validity of plasmacatecholamine estimations. Lancet, 2, 62.

Chidsey, C. A., Braunwald, E., and Morrow, A. G. (1965). Catecholamine excretion and cardiac stores of norepinephrine in congestive heart failure. American fournal of Medicine, 39, 442.
Crout, J. R. (1959). Some spectrophotofluorimetric observations on blood and urine catecholamine assays. Pharmacological Reviews, 11, 296.

Engelman, K., Portnoy, B., and Lovenberg, W. (1968). A sensitive and specific double-isotope derivative method for the determination of catecholamines in biological specimens. American fournal of the Medical Sciences, 255, 259.

Fluck, D. C., Olsen, E., Pentecost, B. L., Thomas, M., Fillmore, S. J., Shillingford, J. P., and Mounsey, J. P. D. (1967). Natural history and clinical significance of arrhythmias after acute cardiac infarction. British Heart fournal, 29, 170.

Forssman, O., Hansson, G., and Jensen, C. C. (1952). The adrenal function in coronary thrombosis. Acta Medica Scandinavica, 142, 44I.

Gazes, P. C., Richardson, J. A., and Woods, E. F. (1959). Plasma catechol amine concentrations in myocardial infarction and angina pectoris. Circulation, 19, 657.

Januszewicz, W., Sznajderman, M., Wocial, B., and Preibiasz, J. (1968). Urinary excretion of free norepinephrine and free epinephrine in patients with acute myocardial infarction in relation to its clinical course. American Heart fournal, 76, 345.

Jewitt, D. E., Mercer, C. J., Reid, D., Valori, C., Thomas, M., and Shillingford, J. P. (1969). Free noradrenaline and adrenaline excretion in relation to the development of cardiac arrhythmias and heart-failure in patients with acute myocardial infarction. Lancet, $1,635$.

McDonald, L., Baker, C., Bray, C., McDonald, A., and Restieaux, N. (1969). Plasma-catecholamines after cardiac infarction. Lancet, 2, 1021 .

Nuzum, F. R., and Bischoff, F. (1953). The urinary output of catechol derivatives including adrenaline in normal individuals, in essential hypertension, and in myocardial infarction. Circulation, $7,96$.

Siggers, D. C., Salter, C., and Toseland, P. A. (I970). A double isotope dilution method for differential determination of adrenaline and noradrenaline in plasma. Clinica Chimica Acta, 30, 373.

Udenfriend, S. (1959). Survey of chemical and physical methods for measuring catecholamines. Pharmacological Reviews, II, 252.

Valori, C., Thomas, M., and Shillingford, J. (1967). Free noradrenaline and adrenaline excretion in relation to clinical syndromes following myocardial infarction. American fournal of Cardiology, 20, 605.

Vendsalu, A. (1960). Studies on adrenaline and noradrenaline in human plasma. Acta Physiologica Scandinavica, 49, Suppl. 173.

Wallace, A. G. (1968). Catecholamine metabolism in patients with acute myocardial infarction. In Acute Myocardial Infarction, pp. 237-242. Ed. by D. G. Julian and M. F. Oliver. E. and S. Livingstone, Edinburgh. 